\title{
Transverse Mode Selection in Vertical-Cavity Surface-Emitting Lasers with Optical Injected Signal
}

\author{
M. S. Torre, A. Valle, and L. Pesquera
}

\begin{abstract}
The transverse mode selection induced by optical injection in a VCSEL emitting in two transverse modes is analyzed from a theoretical point of view. Different pairs of transverse modes with parallel or orthogonal polarizations are considered. In general, we show that transverse mode selection can be achieved when the two modes have parallel or orthogonal polarizations. The selection is not possible only when both modes have parallel polarizations and the mode with the lowest threshold current has a lower injection rate than the other mode. Different forms of the selection region in the injected powerinjection current plane appear depending on the polarization of the transverse modes. When both transverse modes are orthogonally polarized the mode with the lowest threshold is selected when the injected power surpasses a value that depends on the injection current. However when both modes have parallel polarizations the selection region has a completely different shape because the selection of the mode is no longer possible if the injected power or the injected current are large enough. We also show that the modal selection is favoured as the frequency of the injected light becomes smaller than the frequency of the mode to be selected.
\end{abstract}

Index Terms-Optical injection, injection locking, transverse modes, vertical-cavity surface-emitting lasers (VCSELs),

\section{INTRODUCTION}

$\mathrm{E}^{\mathrm{x}}$ XTERNAL optical injection in semiconductor lasers has been a subject of interest for many years [1]-[2]. Injection locking is a technique commonly employed to reduce the frequency chirp [3], to enhance the spectral stability [4], to suppress the laser noise [5] and to improve the laser intrinsic frequency response [6]. Such studies were extended to vertical-cavity surface-emitting lasers (VCSELs) [7]-[8] which

Manuscript received February 25, 2008. This work was supported in part by the the Ministerio de Educación y Ciencia (MEC), Spain, under project TEC2006-13887-C05-02/TCM. The work of M. S. Torre was supported in part by the CONICET Grant PIP 6474, Argentina.

M. S. Torre is with the Instituto de Física "Arroyo Seco", U.N.C.P.B.A., Pinto 399 (7000) Tandil, Argentina (e-mail: marita@exa.unicen.edu.ar)

A. Valle is with the Instituto de Física de Cantabria, Consejo Superior de Investigaciones Científicas (CSIC), Universidad de Cantabria, E-39005 Santander, Spain (Phone: (34) 942 201465. Fax: (34) 942 200935. e-mail: valle@ifca.unican.es).

L. Pesquera is with the Instituto de Física de Cantabria, Consejo Superior de Investigaciones Científicas (CSIC), Universidad de Cantabria, E-39005 Santander, Spain (e-mail: pesquerl@ifca.unican.es). present significant advantages over their edge-emitting counterparts, including a low threshold current, singlelongitudinal-mode operation and wafer-scale integrability [9]. VCSELs are also attractive for use in injection locking because of its compactness, low power consumption, and circular output beam [10]-[11]. Besides its fundamental research interest, optical injection in VCSELs can be used in all-optical and reconfigurable optical switches [12], to achieve polarization control [13], and to obtain rich nonlinear dynamics [14]-[18]. Those dynamics include chaotic behavior that could be used for data encryption applications [19]. Recent experimental work has also shown the possibility of using optical injection in VCSELs for achieving transverse mode selection [10], [20] or for performing several optical signal processing functions like all-optical inversion [10],[21] and all-optical regeneration [22]. Those applications are based on the transverse mode switching in VCSELs induced by the external optical injection.

Free-running VCSELs usually emit in several transverse modes. That multimode emission is attributed to Spatial Hole Burning (SHB) effects [9],[23]-[24]. Emission in different polarization modes can also be found in free-running VCSELs. In fact, small changes of the device temperature or injection current may result in a polarization switching (PS) between its two linear polarizations [25]-[28]. Optical injection experiments have been usually performed by using two different configurations. In the first one, the "orthogonal optical injection" configuration, linearly polarized light is injected with a polarization that is orthogonal to the linear polarization of the free-running VCSEL [16]-[18],[29]-[30]. In the second one, the "parallel optical injection", both the VCSEL and the injected light polarizations are parallel [7]-[8], [11],[14]-[15],[20],[31]-[33]. Experimental results using parallel injection in a single-transverse mode VCSEL show that injection locking show a dependence on the injected power and frequency detuning that is similar to the one found in edge-emitting devices [31]. Recent experimental studies using multimode VCSELs have shown that the fundamental transverse mode can be selected by using optical injection that is parallel to the linear polarization of that mode [11],[20]. That selection process depends on the polarization of the transverse modes of the free-running VCSEL. The case in which the VCSEL emits in two transverse modes has been 
considered in recent experimental works [11],[20]. Selection of the fundamental transverse mode can be achieved when both modes are orthogonally polarized [11],[20]. However different results have been obtained when the VCSEL has two parallel-polarized modes because the selection of the fundamental mode was achieved in Ref. [11] while in Ref. [20] that selection was not possible. The corresponding theoretical works have focused on the possibility of selecting the fundamental transverse mode, $\mathrm{LP}_{01}$. Two sets of modes have been considered. The first one corresponds to the competition between $\mathrm{LP}_{01}$ and the first higher-order mode, $\mathrm{LP}_{11}$ [14]. The second one is related to the competition between $\mathrm{LP}_{01}$ and $\mathrm{LP}_{02}$ modes [32]. In both cases the selection of the $\mathrm{LP}_{01}$ mode is obtained if the linear polarization of both transverse modes is orthogonal [14],[32]. The case where the polarization of both transverse modes is parallel has only been studied for the competition between $\mathrm{LP}_{01}$ and $\mathrm{LP}_{02}$ modes [32]. In that case the laser does not turn into a single $\mathrm{LP}_{01}$ mode when increasing the injection power [32], in agreement with Ref. [20]. The variety of experimental results [11],[20], suggests that the selection of the $\mathrm{LP}_{01}$ mode when both modes have parallel polarizations can depend on the specific set of modes in which the VCSEL is emitting. In particular, the case of the selection of the $\mathrm{LP}_{01}$ mode when both, $\mathrm{LP}_{01}$ and $\mathrm{LP}_{11}$ modes, have parallel polarizations has not been considered yet. Moreover, the study of the selection for any possible pair of modes, with parallel or orthogonal polarizations, would be desirable to shed more light on the interpretation of the experimental results [11],[20].

In this paper we perform the previously suggested analysis by using the theoretical model used in Refs. [14],[32] that was orginally developed in Ref. [24]. The model is presented in section II. The modal selection processes when the transverse modes have orthogonal and parallel polarizations are analyzed in Sections III and IV, respectively. In the case of transverse modes with parallel polarizations we will identify the injection parameters - injected power and frequency detuning - and the injected current values that lead to an optimum selection of the transverse mode with the lowest threshold current. Finally, Section V contains a summary and the conclusions.

\section{MODEL}

The model utilised [14],[24],[32] incorporates both spatial dependence of carrier and optical field profiles. The model can be used to describe dynamics in VCSELs provided that the modal profiles are determined by an effective waveguide structure. This waveguide can be due to either thermal lensing, an oxide layer or a mesa structure. This model based on modal expansion has been compared with a full spatiotemporal model [34]. The two descriptions agree when the effective index step is greater than the contribution due to the carrier-induced refractive index [34]. In this work we take several effective index step values of the waveguide, $\Delta n=0.1, \Delta n=0.05$, and $\Delta n=0.01$, such that the previous condition is satisfied [34].
The electrical field, $\vec{E}(r, t)$, is expanded in terms of the electrical fields corresponding to the transverse modes that are supported by the laser waveguide. The model describes a cylindrical weakly-index guided VCSEL as illustrated in Fig. 1 of [24]. The appropriate transverse modes for the assumed VCSEL structure are conventionally denoted as the $\mathrm{LP}_{\mathrm{mn}}$ modes. The electrical field is expressed as

$\vec{E}(r, t)=\frac{1}{2}\left(\psi_{1}(r) E_{1}(t) e^{-i \omega_{1} t} \vec{i}+\psi_{2}(r) E_{2}(t) e^{-i \omega_{2} t} \vec{j}\right)+c c$

where $E_{i}(t), \psi_{i}(r)$ and $\omega_{i}$ are, respectively, the complex field amplitude, electrical field profile and frequency of the $i$ transverse mode $(i=1,2)$, while $r$ is the modulus of the spatial vector in the active layer. We assume operation in just two transverse modes. The mode 1 is the one with the lowest threshold current. We will considered different pairs of lasing transverse modes to gain insight on how their spatial overlap affects the transverse mode selection process induced by the optical injection. The Eq. (1) describes the situation in which the VCSEL has two orthogonal transverse modes. The complementary situation in which both transverse modes have parallel polarizations is also considered when changing the $\vec{j}$ vector by the $\vec{i}$ vector in Eq. (1).

The dynamical evolution of the complex field amplitude of each transverse mode is governed by the following rate equation:

$$
\begin{aligned}
\frac{d E_{i}(t)}{d t}= & \frac{1-\mathrm{i} \alpha}{2}\left[v_{g} \Gamma g_{i}(t)-\frac{1}{\tau_{p, i}}\right] E_{i}(t)+ \\
& +\sqrt{\frac{\beta \pi d \int_{0}^{a} N(r, t) r d r}{\tau_{s}}} \xi_{i}(t)+\kappa_{i} \sqrt{P_{i n j}} e^{-i \Delta \omega_{i} t}
\end{aligned}
$$

where $g_{i}(t)$ and $\tau_{p, i}$ are the modal gain and photon lifetime, respectively, of the $i$-mode, $v_{g}$ is the group velocity, $\Gamma$ is the longitudinal confinement factor, $\alpha$ is the linewidth enhancement factor, $a$ is the waveguide radius, $d$ is the width of the active region, $\tau_{s}$ is the nonradiative carrier lifetime and $\beta$ is the spontaneous emission factor. The modal gain of the $i$ mode is given by

$$
g_{i}(t)=\frac{\int_{0}^{\infty} \psi_{i}^{2}(r) g(r, t) r d r}{\int_{0}^{\infty} \psi_{i}^{2}(r) r d r}
$$

where $g(r, t)$ is the local gain and $\psi_{i}(r)$ are obtained by solving the Helmholz equation [24]. We consider a local gain of the form

$$
g(r, t)=\frac{A_{0}\left(N(r, t)-N_{t}\right)}{1+\varepsilon \sum_{i}\left|E_{i}(t)\right|^{2}}
$$

where $N(r, t)$ is the carrier density, $N_{t}$ is the carrier density at 
transparency, $A_{0}$ is the gain coefficient and $\varepsilon$ is the gain saturation parameter. The modal gain represents the degree of spatial overlap between the mode intensity profile and the carrier distribution. The fluctuating nature of the spontaneous emission is included in our model since $\xi_{i}(t)$ is a complex Gaussian term of zero mean and time correlation given by $<\xi_{i}(t) \xi_{j}\left(t^{\prime}\right)>=2 \delta_{i j} \delta\left(t-t^{\prime}\right)$. The last term of Eq. (2) accounts for optical injection. The mode that we want to select by using external optical injection is the one with the lowest threshold current. Then we choose a linearly polarized injected light that is parallel to the mode 1 ( $\vec{i}$ direction). If both transverse modes have parallel polarizations the light is injected in both modes and then both $\kappa_{1}$ and $\kappa_{2}$ are greater than zero, where $\kappa_{i}$ is the injection parameter in the $i$-mode given by [2]

$$
\kappa_{i}=\frac{1}{\tau_{L}}\left(\frac{1}{r_{2}}-r_{2}\right) \sqrt{\eta_{i n j, i}}
$$

where $r_{2}$ is the output-mirror field reflectivity, $\tau_{L}$ is the VCSEL round-trip time and $\eta_{i n j, i}$ is the the coupling efficiency of the injected light to the $i$-transverse mode optical field in the laser cavity. Different optical field overlaps between the injected light and the $i$-transverse mode can be taken into account by considering the appropriate value of $\eta_{i n j, i}$. When both transverse modes are orthogonally polarized, the light is

TABLE I

DEVICE AND MATERIAL PARAMETERS

\begin{tabular}{lll}
\hline \hline \multirow{2}{*}{ Symbol } & \multicolumn{1}{c}{ Quantity } & \multicolumn{1}{c}{ Value } \\
\hline$a$ & Radius of the core region & $3 \mu \mathrm{m}$ \\
$L$ & Length of the cavity & $1 \mu \mathrm{m}$ \\
$d$ & Active region thickness & $0.03 \mu \mathrm{m}$ \\
$s$ & Radius of the injection current & $3 \mu \mathrm{m}$ \\
$D$ & Diffusion coefficient & $5 \mathrm{~cm}^{2} \mathrm{~s}^{-1}$ \\
$\tau_{S}$ & Non radiative carrier lifetime & $2 \mathrm{~ns}$ \\
$\tau_{p, i}$ & Photon lifetime & $1.5 \mathrm{ps}$ \\
$A_{0}$ & Gain coefficient & $3 \times 10^{-16} \mathrm{~cm}^{2}$ \\
$N_{t}$ & Carrier density at transparency & $1.33 \times 10^{18} \mathrm{~cm}^{-3}$ \\
$\alpha$ & Linewidth enhacement factor & 3 \\
$\beta$ & Spontaneous emission factor & $2 \times 10^{-5}$ \\
$\Gamma$ & Longitudinal confinement factor & 0.06 \\
$\kappa_{l}$ & Injection parameter & $1.1 \times 10^{11} \mathrm{~s}^{-1}$ \\
$\varepsilon$ & Gain saturation parameter & $3 \times 10^{-6}$ \\
& &
\end{tabular}

only coupled in the mode 1 and then $\kappa_{1}>0$ and $\kappa_{2}=0 . P_{i n j}$ is the injected power in units of photon number and $\Delta \omega_{l}=\omega_{i n j}-\omega_{l}$ is the frequency detuning of the injected signal from the frequency of the $i$-mode. Results in this work are given in terms of the detuning $\Delta \omega=\omega_{i n j}-\omega_{1}$ with respect to the lowest threshold mode.

The evolution of the carrier density profile, $N(r, t)$, is given by the carrier continuity equation applied to the active region:

$$
\begin{aligned}
\frac{\partial N(r, t)}{\partial t}=\frac{D}{r} & \frac{\partial}{\partial r}\left(r \frac{\partial N(r, t)}{\partial r}\right)-\frac{N(r, t)}{\tau_{s}}+\frac{j(r)}{e d} \\
& -\sum_{i} a_{i} \psi_{i}^{2}(r) g(r, t)\left|E_{i}(t)\right|^{2}
\end{aligned}
$$

where $D$ is the diffusion coefficient, $j(r)$ is the current density injected in the active region, $e$ is the electron charge, and $a_{i}=v_{g} \Gamma\left(2 \pi d \int_{0}^{\infty} \psi_{i}^{2}(r) r d r\right)^{-1}$. We consider a uniform injection of current over a disk of radius $s$ and then, $j(r)=j$ if $r<s$, and $j(r)=0$ elsewhere. The evolution of the electrical field is found by integrating Eqs. (2) and (6). The meaning and numerical values of the parameters used in this work are detailed in Table I. The obtained threshold current density, $j_{t h}$, is $1.77 \mathrm{kA}^{\mathrm{cm}} \mathrm{cm}^{2}$, that corresponds to a $0.5 \mathrm{~mA}$ threshold current.

\section{TRANSVERSE MODE SELECTION FOR ORTHOGONALLY POLARIZED MODES}

We first consider the effect of the optical injection on a VCSEL that is emitting in the orthogonally polarized fundamental transverse mode $\mathrm{LP}_{01}$ and the first higher order mode, $\mathrm{LP}_{11}$. We study this pair of modes because their excitation is one of the most commonly found situations. The mode with the lowest threshold is the $\mathrm{LP}_{01}$ mode [24]. The light is injected at the frequency of the fundamental mode and then $\Delta \omega=0$. The temporal evolution of the power of the two transverse modes is shown in Fig. 1 for several values of the injected power when the injected current is instantaneously changed from a below threshold value to $2 j_{t h}$ at $t=0$. We illustrate in Fig. 1(a)-(b) the power and the modal gain of each
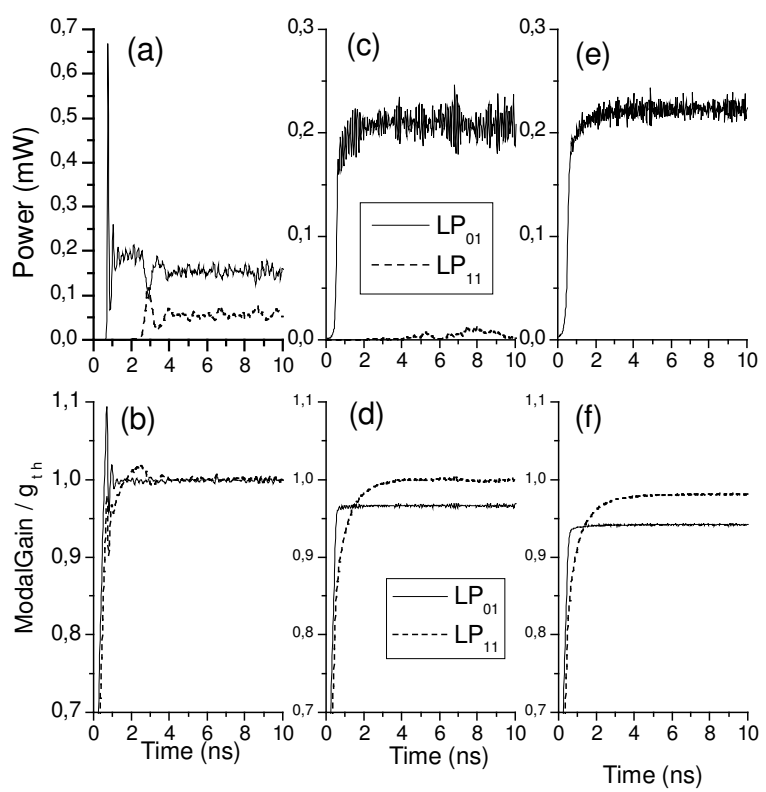

Fig. 1. Temporal series of the power and modal gains of $\mathrm{LP}_{01}$ and $\mathrm{LP}_{11}$ modes at zero frequency detuning, $\Delta n=0.01$, and at injected power of (a)(b), $-40 \mathrm{~dB},(\mathrm{c})-(\mathrm{d}),-10 \mathrm{~dB}$ and (e)-(f), $-5 \mathrm{~dB}$. 
transverse mode for the case of a very weak injected power.

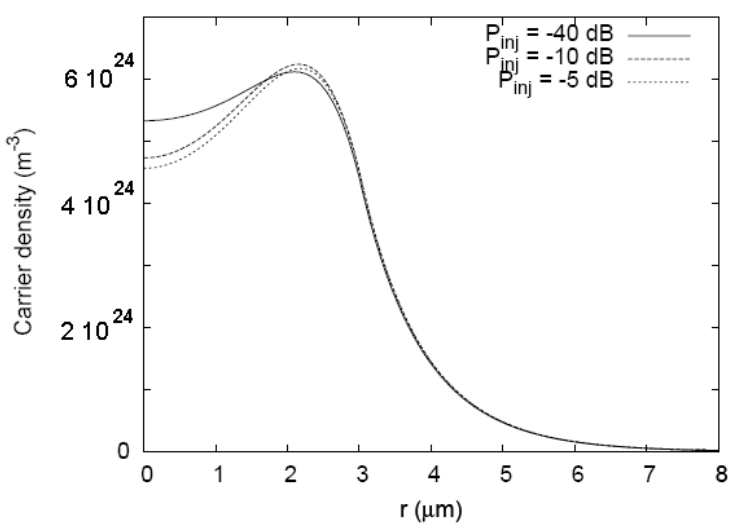

Fig. 2. Carrier density at $t=10 \mathrm{~ns}$ for different injected powers at zero frequency detuning and $\Delta n=0.01$.

The modal gain is normalized to the threshold gain with no injection, $g_{t h}=1 /\left(v_{g} \Gamma \tau_{p}\right)$. The injected power is given in $\mathrm{dBs}$ with respect to the power of the solitary laser that corresponds to the on-state current.

The laser behaves as a solitary VCSEL: there is emission in the two modes because both reach the threshold gain, that is basically unaffected by the optical injection (see Fig. 1(b)).

The situation changes when increasing $P_{i n j}$ (see Fig. 1(c))

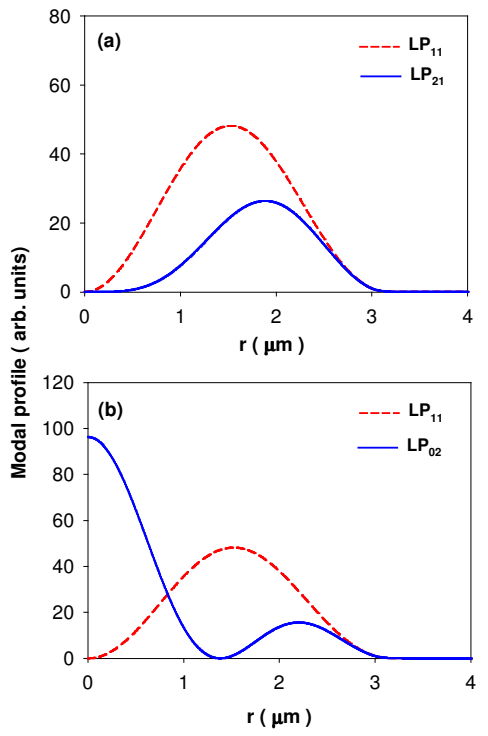

Fig. 3. Power of transverse modes as a function of the distance to the center of the VCSEL for the case of maximum (a) and minimum (b) spatial overlap when $\Delta n=0.05$.

because the threshold gain of the fundamental mode decreases due to the injection while the threshold gain of the $\mathrm{LP}_{11}$ mode remains unaffected (see Fig. 1(d)). We also show in Fig. 2 the carrier densities at the stationary state for the three injection levels considered in Fig. 1. When $P_{i n j}$ is increased from -40 to $-10 \mathrm{~dB}$, both the power of the $\mathrm{LP}_{01}$ mode and the stimulated recombination of carriers near $r=0$, increase. In this way a deeper hole in the carrier density is produced near the center of the device (see Fig. 2). $\mathrm{LP}_{11}$ mode is still lasing since its modal gain is still able to reach the threshold gain but its power has decreased (see Fig. 1(c)). A complete selection of the $\mathrm{LP}_{01}$ mode occurs when increasing $P_{i n j}$ because the modal gain of the $\mathrm{LP}_{11}$ mode is no longer able to reach the threshold gain (see Figs. 1(e)-(f)). This occurs since the carrier density at high

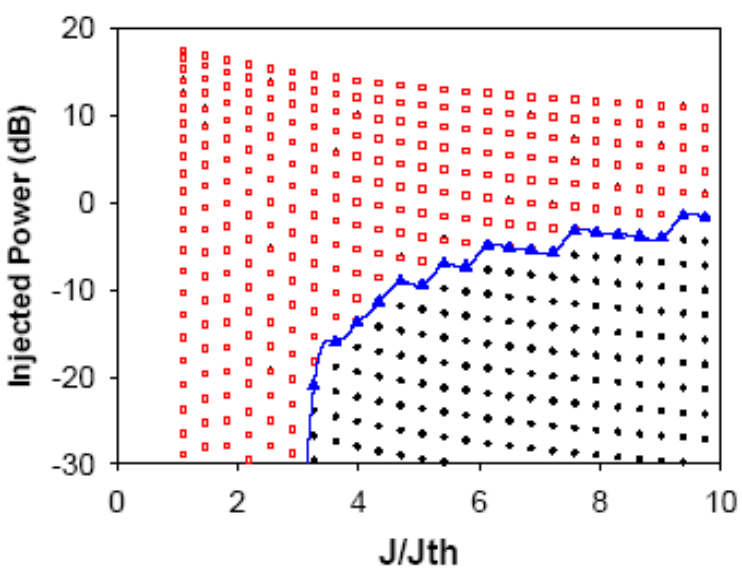

Fig. 4. Region of selection of the $\mathrm{LP}_{11}$ mode (red open squares) when the free-running VCSEL emits in the $\mathrm{LP}_{11}$ and $\mathrm{LP}_{21}$ modes. In this figure $\Delta n=0.05$ and $\Delta \omega=0$.

levels of $P_{i n j}$ decreases at all positions (compare results for $P_{i n j}$ $=-10 \mathrm{~dB}$ and $P_{i n j}=-5 \mathrm{~dB}$ in Fig. 2).

In order to analyze the effect of the spatial overlap between modes we now consider the selection process for another pair of orthogonally polarized transverse modes, the $\mathrm{LP}_{11}$ and $\mathrm{LP}_{21}$ modes. The mode with the lowest threshold current is the $\mathrm{LP}_{11}$ mode. We have chosen those modes because they are the modes that have a maximum spatial overlap. This can be seen in Fig. 3 (a) where the transverse profile of the power of those modes has been plotted. The spatial overlap is characterized by the following quantity [35]:

$$
s=\frac{\left\langle\psi_{1}^{2} \psi_{2}^{2}\right\rangle^{2}}{\left\langle\psi_{1}^{4}><\psi_{2}^{4}\right\rangle}
$$

that has a value of 0.877 for the previous modes ( $s$ values of 0 and 1 mean minimum and maximum spatial overlap).

We characterize in Fig. 4 the selection process for a wide range of injection currents. The red open squares represent the values of $P_{i n j}$ and injection currents where the selection of the $\mathrm{LP}_{11}$ mode has been achieved. We consider that a given mode has been selected when its power represents more than $99 \%$ of the total power. Fig. 4 shows that once the injected mode 
has been selected an increase of $P_{i n j}$ does not lead to the appearance of the other mode. The curve that separates the selection and no selection regions (quenching power [14]) is also shown in that figure.

The region of selection depends also on the specific set of chosen modes. We show in Fig. 5 the quenching power for the $\mathrm{LP}_{11}-\mathrm{LP}_{21}$ and $\mathrm{LP}_{11}-\mathrm{LP}_{02}$ pairs. These two pairs correspond to the cases of maximum and minimum spatial overlap, respectively (see Fig. 3). In both cases the mode with the lowest threshold is the $\mathrm{LP}_{11}$ mode. The quenching power of the $\mathrm{LP}_{11}-\mathrm{LP}_{02}$ modes is much larger than the corresponding to the $\mathrm{LP}_{11}-\mathrm{LP}_{21}$ pair. The reason lies in the low spatial overlap between the $\mathrm{LP}_{11}$ and $\mathrm{LP}_{02}$ modes $(s=0.208)$. In fact, those modes are the ones with minimum spatial overlap, as it is illustrated in Fig. 3(b). Maximum (minimum) spatial overlap means maximum (minimum) competition between modes for the charge carriers. When the competition is large $\left(\mathrm{LP}_{11}-\mathrm{LP}_{21}\right.$ pair) a small amount of injected power is enough to make the $\mathrm{LP}_{11}$ mode to dominate. However, in the case of the $\mathrm{LP}_{11}-\mathrm{LP}_{02}$ pair a much larger injection power is needed to suppress the $\mathrm{LP}_{02}$ mode because that mode is feeding from charge carriers well separated from the carriers that feed the $\mathrm{LP}_{11}$ mode.

\section{TRANSVERSE MODE SELECTION FOR MODES WITH PARALLEL POLARIZATIONS}

This section is devoted to the study of the transverse mode selection due to optical injection when the solitary VCSEL emits in two transverse modes with parallel polarizations. We first consider the case in which the injection rate in both

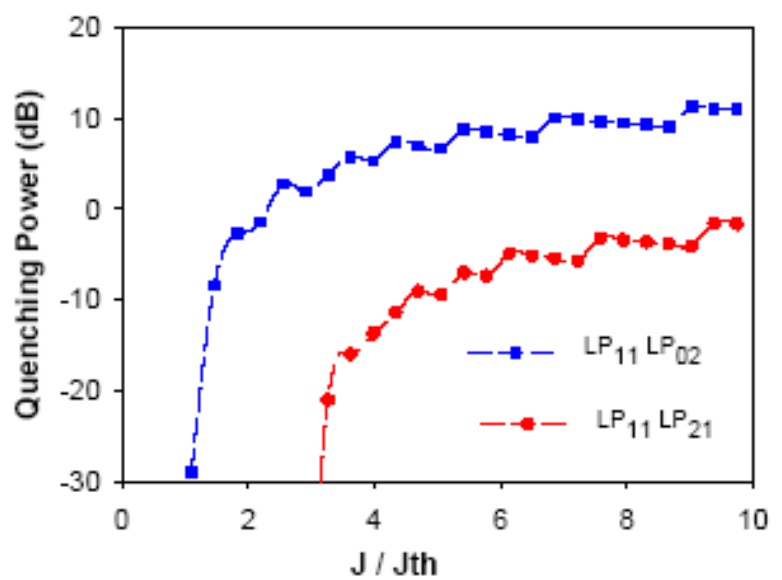

Fig. 5. Minimum power needed to suppress the higher order mode for the case of maximum (red circles) and minimum (blue squares) spatial overlap. In this figure $\Delta n=0.05$ and $\Delta \omega=0$.

transverse modes is equal, $\kappa_{2}=\kappa_{1}$. We show in Fig. 6 (a) the region of selection of the $\mathrm{LP}_{01}$ mode for the case of a VCSEL emitting in the $\mathrm{LP}_{01}-\mathrm{LP}_{11}$ modes. The situation is completely different to the one reported in the previous section. Now selection is only obtained in a small region of the $P_{i n j}$-current
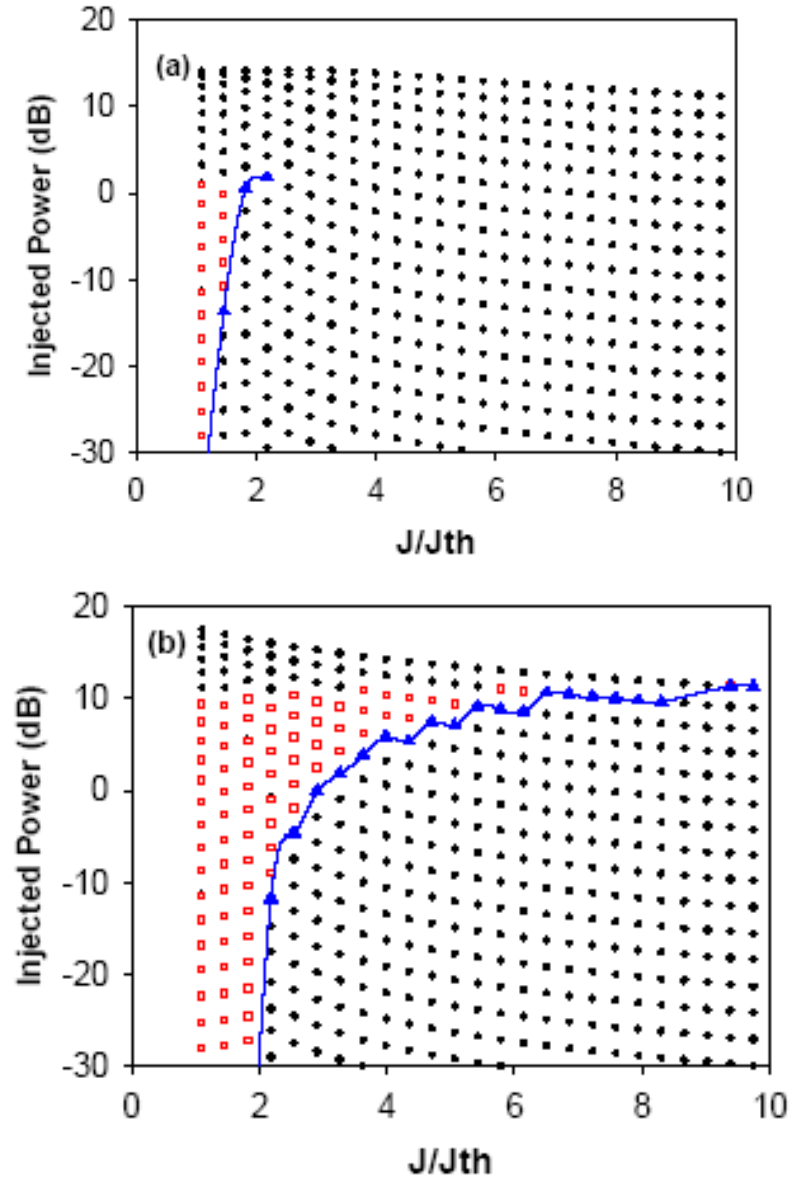

Fig. 6. (a) Region of selection of the $\mathrm{LP}_{01}$ mode (red open squares ) when the VCSEL emits in the $\mathrm{LP}_{01}$ and $\mathrm{LP}_{11}$ modes. (b) Region of selection of the $\mathrm{LP}_{01}$ mode (red open squares) when the VCSEL emits in the $\mathrm{LP}_{01}$ and $\mathrm{LP}_{02}$ modes. Light is injected at the $\mathrm{LP}_{01}$ mode frequency and $\kappa_{2}=\kappa_{1}$. In this figure $\Delta n=0.1$ and $\Delta \omega=0$.

plane. The region of selection has a "tongue" shape. That is due to two new qualitative behaviors. First, selection of the $\mathrm{LP}_{01}$ mode is no longer possible if the current is larger than a certain value. Second, for the values of the current where the $\mathrm{LP}_{01}$ is selected an increase of $P_{i n j}$ leads again to the appearance of the other mode. The area of the selection region increases very much if a different pair of modes is considered. We show in Fig. 6(b) the situation corresponding to the $\mathrm{LP}_{01^{-}}$ $\mathrm{LP}_{02}$ pair. The shape of the selection region is similar to the one in Fig. 6(a) but the mode $\mathrm{LP}_{01}$ is selected in a much larger area of the $P_{i n j}$-current plane.

We have chosen the $\mathrm{LP}_{01}-\mathrm{LP}_{11}$ and $\mathrm{LP}_{01}-\mathrm{LP}_{02}$ pairs to shed light on the previously reported behaviors: experimental and theoretical work on the $\mathrm{LP}_{01}-\mathrm{LP}_{02}$ pair showed that selection of the $\mathrm{LP}_{01}$ mode was not possible [20],[32] while experiments performed by Onishi in a different VCSEL [11] showed that the fundamental transverse mode could be selected. Our results show that the selection of the $\mathrm{LP}_{01}$ mode can be achieved by selecting the appropriate injection current. The parameter region where the $\mathrm{LP}_{01}$ mode is selected depends very much on the transverse modes pair that is chosen. In fact it is easier to 
select the fundamental mode in the case of the $\mathrm{LP}_{01}-\mathrm{LP}_{02}$ pair. Those results are, in principle, in contradiction with the previous theoretical results [32]. That contradiction does not really exists because the injection parameters considered here and in Ref. [32] are different. Until now we have considered that the injection rate in both transverse modes is equal, $\kappa_{2}=\kappa_{1}$. However Ref. [32] considered the situation in which the $\mathrm{LP}_{02}$ mode had a larger injection rate $\kappa_{2}=2 \kappa_{1}$. That is precisely the situation illustrated in Fig. 7. That figure shows that the selection region shrinks very much because the injection favours the $\mathrm{LP}_{02}$ mode more than in Fig. 6(b). Fig. 7 also shows that the selection of the $\mathrm{LP}_{01}$ mode was not obtained in Ref. [32] because their considered currents were always of the order or larger than twice the threshold current. We have also considered the possibility of selecting the $\mathrm{LP}_{01}$ mode when the VCSEL emits in the $\mathrm{LP}_{01}-\mathrm{LP}_{11}$ pair and the injection rate is larger in the $\mathrm{LP}_{11}$ mode $\left(\kappa_{2}=2 \kappa_{1}\right)$. In that case there are not any values of $P_{i n j}$ and the injection current in which the $\mathrm{LP}_{01}$ is selected.

We now discuss the mode selection when different pairs of transverse modes are considered. We show in Fig. 8 the percentage of area in a region of the $P_{i n j}$-current plane (of $10 j_{t h}$ width and $40 \mathrm{~dB}$ height) where the mode with the lowest threshold is selected. We have chosen the pairs with maximum and minimum spatial overlap $\mathrm{LP}_{11}-\mathrm{LP}_{21}, \mathrm{LP}_{11}-\mathrm{LP}_{02}$, and the two pairs illustrated in Fig. 6. The $\mathrm{LP}_{11}-\mathrm{LP}_{21}$ pair is the one in which the selection of the lowest threshold mode is easier. That fact can be attributed to their maximum competition for

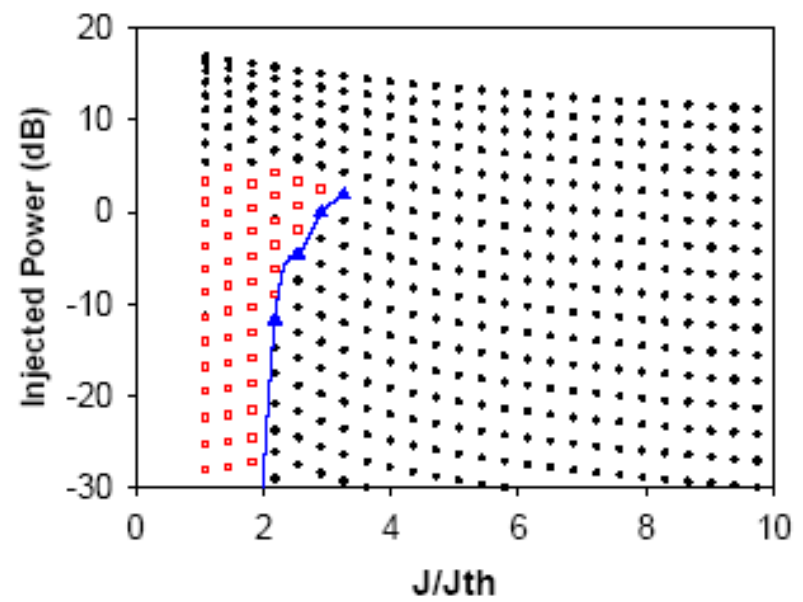

Fig. 7. Region of selection of the $\mathrm{LP}_{01}$ mode (red open squares) when the VCSEL emits in the $\mathrm{LP}_{01}$ and $\mathrm{LP}_{02}$ modes. Light is injected at the $\mathrm{LP}_{01}$ mode frequency and $\kappa_{2}=2 \kappa_{1}$. In this figure $\Delta n=0.1$ and $\Delta \omega=0$.

the carriers due to their maximum spatial overlap $(s=0.877)$. The general trend of having a larger area of selection when the spatial overlap increases is also followed when the $\mathrm{LP}_{01}-\mathrm{LP}_{02}$, and $\mathrm{LP}_{11}-\mathrm{LP}_{02}$ pairs are considered $(s=0.563$, and $s=0.208$, respectively). However, the $\mathrm{LP}_{01}-\mathrm{LP}_{11}$ pair does not follow that trend because their spatial overlap coefficient is $s=0.632$.

Fig 8 also shows the dependence of the area of selection on the detuning between the frequency of the injected light and the frequency of the mode that is selected. The general trend, followed by the $\mathrm{LP}_{01}-\mathrm{LP}_{02}, \mathrm{LP}_{11}-\mathrm{LP}_{02}$, and $\mathrm{LP}_{01}-\mathrm{LP}_{11}$ pairs is given by a monotonous decrease of the selection area as the frequency detuning increases. That means that negative frequency detuning favors the modal selection. Again, an exception to the previous general rule appears when an

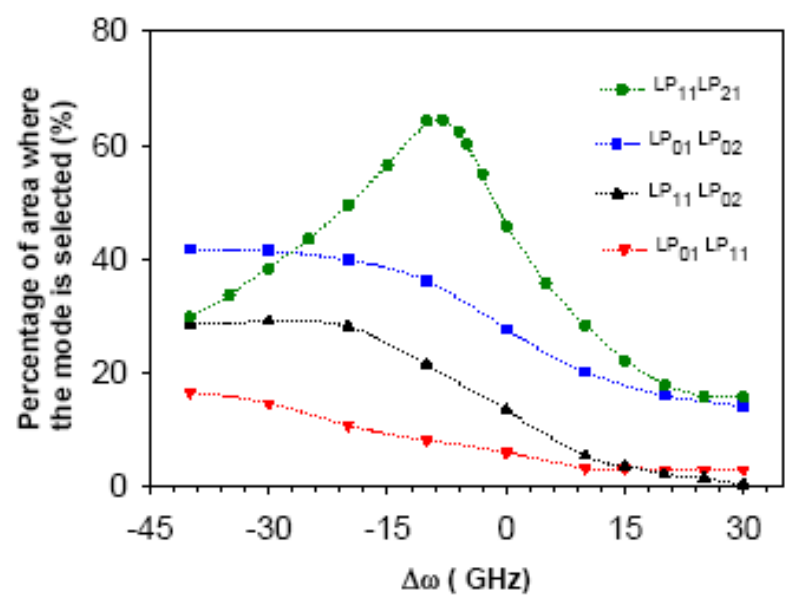

Fig. 8. Percentage of the area where the mode with the lowest threshold current is selected for different pairs of transverse modes. In this figure $\kappa_{2}=\kappa_{1}$ and $\Delta n=0.1$. The transverse modes have parallel polarizations.

specific pair of modes, $\mathrm{LP}_{11}-\mathrm{LP}_{21}$, is considered. The percentage of area where selection of $\mathrm{LP}_{11}$ is achieved is maximum when the frequency detuning is $-9 \mathrm{GHz}$. Then an optimum frequency detuning for mode selection is found when that pair of modes is considered. The asymmetry of all the previous curves can be attributed to the frequency asymmetry induced by the linewidth enhancement factor.

\section{SUMMARY AND CONCLUSIONS}

In this work we have analyzed the transverse mode selection induced by optical injection in a VCSEL that emits in two transverse modes. Attention has been paid to different pairs of transverse modes. Optical injection is applied to select the transverse mode with the lowest threshold current. In general we have shown that the transverse mode selection can be achieved when the two modes have parallel or orthogonal polarizations. We have characterized that selection process in the injected power-injected current plane. When the two transverse modes have orthogonal polarizations, once the injected mode has been selected an increase of the injected power does not lead to the appearance of the other mode. That behavior is obtained for all the values of the injected current. The shape of the selection region completely changes when both modes have parallel polarizations. That region has a "tongue" shape because i) once the injected mode has been selected an increase of the injected power leads again to multimode operation, and ii) the selection of the mode is no longer possible if the current surpasses a certain value. The area of the selection region strongly depends on the considered pair of transverse modes. As a general trend, larger selection 
areas correspond to larger spatial overlaps between modes. We have also identified cases where the region of selection shrinks very much in such a way that the selection is no longer possible. Those cases correspond to situations in which the mode with the lowest threshold current has a lower injection rate than the other mode. In that situation we have recovered the results reported in Ref. [32]. We have also shown that the modal selection is favoured as the frequency of the injected light becomes smaller than the frequency of the mode to be selected.

Finally, the area of the selection region increases with the spatial overlap between modes when they are orthogonally polarized. That trend is not so clear for the case of modes with parallel polarizations. We also note that the selection regions are smaller when the transverse modes have parallel polarizations than when they are orthogonal. In this way selection of a transverse mode induced by optical injection is favoured if both transverse modes of the solitary VCSEL are orthogonally polarized. Further experimental work trying to confirm the results included in this paper is now in progress.

\section{ACKNOWLEDGMENT}

The authors would like to thank Bruno Casal for his preliminary work.

\section{REFERENCES}

[1] R. Lang, "Injection locking properties of a semiconductor laser", IEEE J. Quantum Electron., vol. 18, no. 6, pp. 976-983, 1982.

[2] G. H. M. van Tartwijk, D. Lenstra, "Semiconductor lasers with optical injection and feedback", Quantum Semiclass. Opt., 7, pp. 87-143, 1995.

[3] N. A. Olsson, H. Temkin, R. A. Logan, L. F. Johnson, G. J. Dolan, J. P. van der Ziel, and J. C. Campbell, "Chirp-free transmission over $82.5 \mathrm{~km}$ of single-mode fibers at $2 \mathrm{~Gb} / \mathrm{s}$ with injection locked DFB semiconductor lasers," J. Lightwav. Technol., vol. 3, no. 2, pp. 63-67, Feb. 1985.

[4] P. Gallion, H. Nakajima, G. Debarge, and C. Chabran, "Contribution of spontaneous emission to the linewidth of an injection-locked semicondiuctor laser", Electron. Lett, vol. 22, pp. 626-628, 1995.

[5] K. Iwashita and K. Nakawaga, "Supression of mode partition noise by laser diode light injection," IEEE J. Quantm Electron., vol. 18, no. 10, pp. 1669-1674, Oct. 1982.

[6] X. Meng, T. Chau, and M. C. Wu, "Experimental demonstration of modulation bandwidth enhacement in distributed feedback lasers with external light injection,” Electron. Lett., vol. 34, no. 21, pp. 2031-2032, 1998.

[7] C. H. Chang, L. Chrostowski, and C. J. Chang-Hasnain, "Injection locking of VCSELs," IEEE J. Selec. Top. In Quantum Electron., vol. 9 , no. 5, pp. 1386-1393, Sep-Oct. 2003.

[8] L. Chrostowski, X. Zhao, and C. J. Chang-Hasnain, "Microwave performance of optically injection-locked VCSELs," IEEE Trans. Microw. Theory Tech., vol. 54, no. 2, pp. 788-796, Feb. 2006.

[9] C. J. Chang-Hasnain, J. P. Harbison, G. Hasnain, A.C. von Lehmen, L. T. Florez, and N. G. Stoffel, "Dynamic, polarization and transverse mode characteristics of vertical cavity surface emitting lasers", IEEE J. Quantum Electron, vol. 27, no. 6, pp. 1402-1409, Jun., 1991.

[10] F. Koyama, "Recent advances of VCSEL Photonics", J. Lightwave Technol. Vol. 24, no. 12, pp. 4502-4513, Dec. 2006.

[11] Y. Onishi, F. Koyama, N. Nishiyama, C. Caneau, and C. E. Zah, "Nonlinear optical input-output characteristics of 1.55 microns injection-locked vertical-cavity surface-emitting lasers," Appl. Phys. Lett., vol. 84, no. 17, pp. 3247-3249, 2004.

[12] K. Panajotov, F. Berghmans, M. Peeters, G. Verschaffelt, J. Danckaert, I. Veretennicoff, and H. Thienpont, "Data transparent reconfigurable optical interconnections using polarization switching in VCSELs induced by optical injection," IEEE Photon. Technol. Lett., vol. 11, no. 8, pp. 985-987, Aug. 1999.

[13] S. Bandyopadhyay, Y. Hong, P. S. Spencer, K. A. Shore, "VCSEL polarization control by optical injection," J. Lightwav. Technol., vol. 21, no. 10 , pp. 2395-2404, 2003.

[14] J. Y. Law, G. H. M. van Tartwijk, and G. P. Agrawal, "Effects of transverse mode competition on the injection dynamics of verticalcavity surface-emitting lasers," Quantum Semiclass. Opt., vol. 9, no. 5, pp. 737-747, 1997.

[15] Y. Hong, P. S. Spencer, S. Bandyopadhyay, P. Rees, K. A. Shore, "Polarization resolved chaos and instabilities in a VCSEL subject to optical injection", Opt. Comm., vol. 216, pp. 185-189, 2003.

[16] J. Buesa, I. Gatare, H. Thienpont, K. Panajotov, and M. Sciamanna, "Mapping of the dynamics induced by orthogonal optical injection in vertical-cavity surface-emitting lasers," IEEE J. Quantum Electron, vol. 42, no. 2, pp. 198-207, Feb. 2006.

[17] I. Gatare, J. Buesa, H. Thienpont, M. Sciamanna, and K. Panajotov, "Nonlinear dynamics accompanying polarization switching in verticalcavity surface-emitting lasers with orthogonal optical injection", Appl. Phys. Lett., 88, 101106, 2006.

[18] I. Gatare, M. Sciamanna, M. Nizette, and K. Panajotov, "Bifurcation to polarization switching and locking in vertical-cavity surface-mitting lasers with optical injection", Phys. Rev. A 76, 031803, 2007.

[19] A. Argyris, D. Syvridis, L. Larger, V. Annovazzi-Lodi, P. Colet, I. Fischer, J. Garcia-Ojalvo, C. R. Mirasso, L. Pesquera, and K. A. Shore, "Chaos-based communications at high bit rates using commercial fiber optic links," Nature, vol. 438, no. 7066, pp. 343-346, 2005.

[20] Y. Hong, P. S. Spencer, P. Rees, K. A. Shore, "Optical injection dynamics of two-mode vertical-cavity surface-emitting semiconductor lasers," IEEE J. Quantum Elctron., vol. 38, no. 3, pp. 274-278, 2002.

[21] Y. Onishi, N. Nishiyama, C. Caneau, F. Koyama, and C. E. Zah, "Dynamic behavior of an all-optical inverter using transverse-mode switching in 1.55 microns vertical-cavity surface-emitting lasers", IEEE Photon. Technol. Lett., vol. 16, no. 5, pp. 1236-1238, 2004.

[22] Y. Onishi, F. Koyama, "All-optical regeneration using a vertical-cavity surface-emitting laser with external light injection", IEICE Trans. Electron., vol. E87-C, no. 3, pp. 409-415, 2004.

[23] D. Vakhshoori, J. D. Wynn, G. J. Zydzik, M. Asom, K. Kojima, R. E. Leibenguth, and R. A. Morgan, "Top-surface emitting lasers with $1.9 \mathrm{~V}$ threshold voltage and the effect of spatial hole burning on their transverse mode operation and efficiencies", Appl. Phys. Lett. Vol. 62, no. 13 , pp. $1448-1450,1993$.

[24] A. Valle, J. Sarma, K. A. Shore, "Spatial hole burning effects on the dynamics of vertical-cavity surface-emitting semiconductor lasers," IEEE J. Quantum Electron., vol. 31, no. 8, pp. 1423-1431, Aug. 1995.

[25] K. D. Choquette, R. P. Schneider, K. L. Lear, and R. E. Leibenguth, "Gain-dependent polarization properties of vertical-cavity lasers," IEEE J. Selec. Topics Quantum Electron., vol. 1, no. 2, pp. 661-666, Mar./Apr. 1995.

[26] J. M. Martín-Regalado, F. Prati, M. San Miguel, and N. B. Abraham, "Polarization properties of vertical cavity surface-emitting lasers, " IEEE J. Quantum Electron., vol. 33, no. 5, pp. 765-783, May 1997.

[27] A. Valle, L. Pesquera, K. A. Shore, "Polarization behavior of birefringent multitransverse mode vertical-cavity surface-emitting lasers," IEEE Photon. Technol. Lett., vol. 9, no. 5, pp. 557-559, May 1997.

[28] B. Ryvkin, K. Panajotov, A. Georgievski, J. Danckaert, M. Peeters, G. Verchaffelt, H. Thienpont, and I. Veretennicoff, "Effect of photonenergy-dependent loss and gain mechanisms on polarization switching in vertical-cavity surface -emitting lasers," J. Opt. Soc. Amer. B, vol.. 16, pp. 2106-2113, 1999.

[29] Z. G. Pan, S. Jiang, M. Dagenais, R. A. Morgan, K. Kojima, M. T. Asom, and R. E. leibenguth, "Optical injection induced polarization bistability in vertical-cavity surface-emitting lasers", Appl. Phys. Lett., vol. 63, pp. 2999-3001, 1993.

[30] A. Valle, I. Gatare, K. Panajotov, and M. Sciamanna, "Transverse mode switching and locking in vertical-cavity surface-emitting lasers subject to orthogonal optical injection,", IEEE J. Quantum Electron., vol. 43, no. 4, pp. 322-333, Apr. 2007.

[31] H. Li, T. L. Lucas, J. G. McInerney, M. W. Wright, and R. A. Morgan, "Injection locking dynamics of vertical-cavity semiconductor lasers 
under conventional and phase conjugate injection," IEEE J. Quantum Electron., vol. 32, no. 2, pp. 227-235, 1996.

[32] M. S. Torre, C. Masoller, and K. A. Shore, "Numerical study of optical injection dynamics of vertical-cavity surface-emitting lasers", IEEE $J$. Quantum Electron., vol. 40, no. 1, pp. 25-30, Jan. 2004..

[33] M. S. Torre, A. Valle, and L. Pesquera, "Polarization and transverse mode behavior of VCSELs under optical injection," Opt. Quantum Electron., vol. 38, pp. 445-465, 2006.

[34] J. Mulet, and S. Balle, "Transverse mode dynamics in vertical-cavity surface-emitting lasers", Phys Rev. A, 66, (5), Art. 053802, 2002.

[35] A. Valle, S. Ortin, L. Pesquera, "Current modulation of multi-transverse mode vertical-cavity surface-emitting lasers", Proceedings of the VII International Conference on Transparent Optical Networks, ICTON 2005, vol. 2, pp. 126-131, 2005.

M. S. Torre received the Licenciada en Física (Ms. Sc.) degree and the $\mathrm{Ph}$. D. degree both from the Universidad Nacional del Centro de la Provincia de Buenos Aires (UNCPBA), Buenos Aires, Argentina.

Her research was primarily in external driven laser physics. From 1995 to 1997, she was a post-Doctoral fellow at the Photonics Technology Department of the ETSI Telecomunicaciones, Universidad Politécnica de Madrid, Madrid, Spain. Her research was in quantum-well semiconductor laser modeling. Since 1988, she has been member of the Physics Institute "Arroyo Seco", UNCPBA. She is currently a Research Professor at the Facultad de Ciencias Exactas of the UNCPBA. and Ajdunto Researcher CONICET ( National Council of Scientific and Technical Investigations) Her research topics include modelling and dynamics of VCSEL, dynamics of semiconductor lasers with external optical feedback, and diffusion effects in semiconductor lasers.

A. Valle was born in Reinosa, Cantabria, Spain, in 1965. He received the M. Sc. and Ph.D. degree in physics from the Universidad de Cantabria, Spain, in 1988 and 1993, respectively.

During 1994 and 1995, he was a postdoctoral fellow at the School of Electronic and Electrical Engineering at the University of Bath, England. In 1996 he joined the Instituto de Física de Cantabria (CSIC-UC). Since 1998 he has been lecturer at the Departamento de Física Moderna at the University of Cantabria, Spain. His research interests are in the areas of vertical-cavity surface-emitting lasers, noise and nonlinear dynamics of semiconductor lasers.

L. Pesquera was born in Vega de Infanzones, León, Spain, in 1952. He received the M.Sc. degree in physics in 1974 from the Universidad de Valladolid, Spain. He was a postgraduate fellow at the Université de Paris VI during 1977-1980. He received the Ph.D. degree in physics in 1980 from the Universidad de Cantabria,Santander, Spain.

In 1980 he joined the Departamento de Física Moderna of the Universidad de Cantabria. Since 1991 he has been Professor of Physics at the Universidad de Cantabria. In 1995 he joined the Instituto de Física de Cantabria (CSICUC). His research work started in the field of stochastic processes applied to Physics and he has made contributions to the foundations of quantum physics, fluctuations in nuclear reactors, disordered systems and laser physics. $\mathrm{He}$ is currently working on the modeling of noise and nonlinear properties of semiconductor lasers and their applications to optical communication systems. 